\title{
The AAC Application on Communication for Children with Autism
}

\author{
Yun Xu \\ College of Education \& Technology, Zhejiang University of Technology, Hangzhou, 310023, China
}

Keywords: AAC, autistic children, communication ability

\begin{abstract}
This paper studies the effect on the Augmentative and Alternative Communication (AAC) System for communication ability of autistic children. Procedures and Methods: Selecting five autistic children as research subjects, and the single subject experimental method was used to train subjects with the AAC system. Results: It's found that the amount and quality of autistic children communication increase significantly. Conclusions: The Augmentative and Alternative Communication System can enhance their communication behavior, and improve their communication ability.
\end{abstract}

\section{Introduction}

The Augmentative and Alternative Communication (AAC) system, putting it another way as "expanding and alternating communication support system", is a method to convey information which can help communication disorders by voice, pictures, et al [1]. Generally it consists of expanding communication systems and alternative communication system, including any communication way to help speaking or writing. In 2005, the American Speech and Hearing Association, ASHA defined that the Augmentative and Alternative Communication system belongs to the field of research, clinical and educational applications, and its purpose is to investigate and if necessary to make up temporary or permanent injury, activity limitations and participation restrictions of people with oral expression or oral comprehension disabilities, which includes speaking and writing modes of communication [2]. The Augmentative and Alternative Communication system is suitable for all ages and different social backgrounds people, who generally need a proper help assist their speaking and writing, to fill them in this capacity defect.

Autism is a serious mental and physical developmental, pervasive and developmental disorder due to brain nervous system disease, and neurodevelopmental disorder which related to perception, emotion, speech, thought, action and behaviour aspects et al. Its symptoms include abnormal social skills, communication skills, behaviour patterns and interests. Communication barrier is one of the three characteristics of autistic children. Nearly half of them are almost completely unable to speak, and rarely use other communication means of gestures, expressions, et al [3]. Although some parts of autistic children have speaking ability, there are tones, semantics, syntax errors or special expression characteristics, such as language appears parrots, metaphorical language, et al, and strangers are difficult to understand the content they want to express and idea, even their teachers and families can't fully understand their content [4]. Defects of communication skills and language abilities hinder interaction and communication between autistic children and social or the environment. Studies have 
shown that $5 \%$ to $10 \%$ people had communication barriers, and almost all autistic children had communication barriers.

From the 1970s beginning to appear, but now the AAC system is still a new technology. In abroad and Taiwan, the AAC system have been many clinical applications, and achieved good effect on the autism treatment and education. As Ganz, Eigsti, Olive, Sigafoos, et al. who used the AAC system to train autistic children, found that they had more communication requirements $[1,4,5,7]$; Guanming Chen who trained autistic children with voice communication board microcomputer, found that the number of their communication behaviour increased, and they were easier to use communication layout [6]. But in mainland of china, there were no study which reported that someone used it to train autistic children.

This study would use the Augmentative and Alternative Communication system to train communication skills of autistic children, and explore its practical effect.

\section{Objects and Methods}

\subsection{Objects}

This study randomly selected five moderate or serious autistic children as the study objects in a special school of Hangzhou. Five objects were diagnosed as autism by related hospital, and had some spoken language, but lacked of effective and initiative communication language; they were 9-10 years old. Their action, perception and hearing were no different with normal children; however they were poor in cognitive ability, language comprehension, and language skills.

\subsection{Materials}

The main tool of this experiment is the Augmentative and Alternative Communication system designed for autistic children. The content of design includes basic vocabulary, living language and social communication language in this experiment. Experiment uses a digital camera with video capabilities to record the trail process of baseline, intervention and maintaining period. Because the digital camera is compact and lightweight, it couldn't be found easily by objects. So it can actually record the experimental process.

\subsection{Experimental design}

The single subject experimental method was used as research measure. During the experiment design of $\mathrm{A}-\mathrm{B}-\mathrm{A}$, the first $\mathrm{A}$ is baseline period in which only observing and recording the behavior of subjects, without doing any training interventions; the B refers to the intervention period, namely training the subjects and making relevant records; the second $\mathrm{A}$ is the maintenance period in which observing the subjects behavior after withdrawing the intervention. The experiment continued for 3 months, 15 minutes each time and twice a week.

Intervention process performed daily language exchanges between director and subjects in this study. The Augmentative and Alternative Communication system presents the options subjects may answered, then director waited subjects for answering questions by clicking on the system screen. If the subjects gave the correct response, continuing the next course content; If not, director shows the contents of the system screen through spoken language, gestures or body; if the subjects have not yet given the correct response, director helps the subjects complete teaching content with pressing the system screen. 


\subsection{Statistical analysis}

The experiment used SPSS 17.0 software to analysis trail statistical. In order to know the trial reliability, inviting an observer to record all communication behavior with no interfering at the third time of intervention period. From consistency check between researcher and observer's record, it was concluded that the consistency was 0.96 .

\section{Results}

The experimental data of five subjects is shown in table 1 .

Table 1: The training results

\begin{tabular}{|c|c|c|c|c|c|c|}
\hline Subject & Period & $\mathrm{n}$ & $\mathrm{X}$ & $\mathrm{C}$ & $\mathrm{Sc}$ & $\mathrm{Z}$ \\
\hline & baseline & 6 & 0 & - & 0.34 & - \\
\hline No.1 & intervention & 12 & 4.67 & 0.23 & 0.27 & 1.07 \\
\hline & maintenance & 8 & 5.00 & 0.21 & 0.31 & 0.67 \\
\hline No.2 & intervention & 12 & 4.92 & 0.29 & 0.26 & 1.09 \\
\hline & maintenance & 8 & 4.13 & 0.49 & 0.31 & 1.60 \\
\hline & baseline & 6 & 3.17 & -0.24 & 0.34 & -0.70 \\
\hline No.3 & intervention & 12 & 9.33 & 0.80 & 0.26 & 3.01 \\
\hline & maintenance & 8 & 9.38 & 0.32 & 0.32 & 1.03 \\
\hline & baseline & 6 & 7.17 & -0.06 & 0.33 & -0.17 \\
\hline No.4 & intervention & 12 & 9.33 & 0.80 & 0.26 & 3.01 \\
\hline & maintenance & 8 & 9.38 & 0.32 & 0.31 & 1.03 \\
\hline & baseline & 6 & 2.50 & 0 & 0.34 & 0 \\
\hline No.5 & intervention & 12 & 4.50 & 0.56 & 0.26 & 2.11 \\
\hline & maintenance & 8 & 3.50 & 0.42 & 0.31 & 1.35 \\
\hline
\end{tabular}

Note: the $\mathrm{n}$ represents the number of experiment; the $\mathrm{X}$ represents the average amount of communication, the $\mathrm{C}, \mathrm{Sc}$, and $\mathrm{Z}$ are the $\mathrm{C}$ statistic.

From table 1, it can be known the communication amount of the first subject was 0 at baseline period. After receiving the AAC system during intervention period, there had a significant increase on communication amount and behavior that the average amount was from 0 to 4.67 times. At maintenance period, the amount of communication was 5.00 on an average, and the $\mathrm{C}$ statistic data showed $\mathrm{C}$ was 0.21 ; Compared $\mathrm{C}$ was 0.28 in intervention period, there was a decreasing trend. It indicated that the communication behavior of maintenance period tended to be stable and intervention effect was persistent. 
The communication amount of the second subject was also 0 at baseline period, namely no communication behavior. Through training of the AAC system at intervention period, the communication amount increased from 0 to 4.90. It showed that communication behavior of second subject had increased significantly after using the AAC system. From table 1, it can be seen the training effect was kept at maintenance period compared to intervention period. The $\mathrm{C}$ statistics showed that communication behavior amount tended to stabilize.

The third subject's communication amount had a stable trend, the $\mathrm{C}$ statistics was -0.24 and $\mathrm{Z}$ was -0.70 at baseline period. After using the AAC system, the average number of communication was from 3.167 to 9.33 , and the $C$ statistics rose from -0.24 to 0.80 at intervention period. It showed an obvious intervention effect, and the communication amount was on the rise. At maintenance period, the average amount of communication behavior was 9.38, and the $\mathrm{C}$ statistics was 0.32 . Compared to the intervention period, it didn't reach a notable level. It made clear that the effect of interventional training persisted at maintaining period.

The communication amount of the forth subject was 7.17 on the average at baseline period and the $\mathrm{C}$ statistical was -0.06 . So there was no significant difference and had a stable state. During intervention period, the $\mathrm{C}$ statistics showed a clear upward trend from -0.06 to 0.80 . At maintaining period, the $\mathrm{C}$ statistics decreased from 0.80 to 0.32 and the average amount of communication maintained at about 9. Thus the data showed a steady trend, meanwhile the effect of intervention period persisted.

The fifth subject's communication amount was 2.50 and the $\mathrm{C}$ statistic was 0 at baseline period. There was a stable trend. After receiving training intervention, the communication behavior increased from 2.50 to 4.50 and the $C$ statistic also increased from 0 to 0.56 at intervention period. The data showed a trend of rising. At maintaining period, although the average amount of communication decreased from 4.50 to 3.50 , the $\mathrm{C}$ statistic was 0.42 which showed stabilization.

From the maintaining period data of five subjects it can be learned, compared to the baseline period, every subject's communication behavior was increased, the rising trend was obvious, and there had a significant difference; Compared to intervention period, the amount of subjects' communication behavior rose, or fell, but the global trend was stable with no significant difference. It can be inferred that, through training five autistic children by the AAC system, the amount of their communication behavior had improved. And after withdrawing training, the effect had been kept.

From the changes of five subjects about communication behavior amount, they had a significant increase after using the AAC system; at the end of the intervention, the subjects could keep intervention training effect; several months later after ending the training, the effect of using AAC system can still be maintained.

\section{Discussions}

In this experiment, the communication amount and desire of the subjects who used the Augmentative and Alternative Communication system had increased significantly. Application results show that the AAC system is feasible on autistic children [7]. After receiving the training of the AAC system and intervening, the communication amount of five subjects had increased significantly, the average number of communication shows an improvement compared to the baseline with no intervening, and the statistical results showed that it reached a significant level. After withdrawing the intervention, the effect of training can be maintained. The finding showed that after receiving the intervention training, the number of autistic children communication behavior had increased significantly. From the research results, it can be found that the AAC system promoted subjects communication with others and increased their power to communicate by sound and cartoons et al, moreover, the subjects actively participated in the communication, made a breakthrough on their communication ability, and enhance their presentation skill $[5,7]$.

After emerging and used in the last century seventy's, the Augmentative and Alternative Communication system achieved a good effect in the field of health care and education. As an expanding and alternating communication aids, it largely solves the problem that person with 
language barriers cannot normal communicate with others, but also provides a process of learning communication skills. It enhances the communication and expression skill of communication disorders and helps the communication barriers better adapt to society. So it is said the AAC system can be widely used in special education.

\section{Acknowledgements}

The research work was under the support of the National Social Science Special Project "The interdisciplinary research of early detection, intervention and education for autistic children (12\&ZD229)”.

\section{References}

1. Jennifer B. Ganz, Theresa L. Earles-Vollrath, \& Rose A. Mason, et al., An aggregate study of single-case research involving aided AAC: Participant characteristics of individuals with autism spectrum disorders. Autism Spectrum Disorders, 5(4), pp. 1500-1509, 2011.

2. Guanghui Huang, Study on improving spoken language expression ability of students with intellectual disabilities at junior high school by the Augmentative and Alternative Communication system. Hualian: Donghua University, pp. 19-44, 2010.

3. Qiang Chen, Yun $\mathrm{Xu}$, The Augmentative and Alternative Communication System and practical technology, Science Press: Beijing, pp. 13-17, 2011.

4. Inge-Marie Eigsti, Ashley B. de Marchena, \& Jillian M. Schuh, et al., Language acquisition in autism spectrum disorders: A developmental review. Autism Spectrum Disorders, 5(2), pp. 681691, 2011.

5. Melissa L. Olive, Berenice de la Cruz \& Tonya N. Davis, et al., The Effects of enhanced milieu teaching and a voice output communication aid on the requesting of three children with autism. Journal of Autism and Developmental Disorders, 37(8), pp. 1505-1513, 2007.

6. Guanming Chen, Study the effect on teaching strategy of voice communication board microcomputer to enhance the communicative behaviors of low oral autistic students of junior high school. Taipei: Taiwan Normal University, pp. 40-42, 2010.

7. Jeff Sigafoos, Mark O’Reilly \& Sue Seely-York, et al., Teaching students with developmental disabilities to locate their AAC device. Developmental Disabilities, 25(4), pp. 371-383, 2004. 